\title{
Detection of Snore from OSAHS Patients Based on Deep Learning
}

\author{
Fanlin Shen $\mathbb{D}^{1},{ }^{1}$ Siyi Cheng, ${ }^{1}$ Zhu Li, ${ }^{1}$ Keqiang Yue $\mathbb{D},{ }^{1}$ Wenjun Li $\mathbb{D}^{1},{ }^{1}$ and Lili Dai ${ }^{2}$ \\ ${ }^{1}$ Key Laboratory of RF Circuits and Systems, Ministry of Education, Hangzhou Dianzi University, Hangzhou, Zhejiang, China \\ ${ }^{2}$ The Affiliated Hospital of Hangzhou Normal University, Hangzhou, Zhejiang, China \\ Correspondence should be addressed to Wenjun Li; liwenjun@hdu.edu.cn
}

Received 17 August 2020; Revised 29 October 2020; Accepted 30 November 2020; Published 12 December 2020

Academic Editor: Jiafeng Yao

Copyright $\odot 2020$ Fanlin Shen et al. This is an open access article distributed under the Creative Commons Attribution License, which permits unrestricted use, distribution, and reproduction in any medium, provided the original work is properly cited.

Obstructive sleep apnea-hypopnea syndrome (OSAHS) is extremely harmful to the human body and may cause neurological dysfunction and endocrine dysfunction, resulting in damage to multiple organs and multiple systems throughout the body and negatively affecting the cardiovascular, kidney, and mental systems. Clinically, doctors usually use standard PSG (Polysomnography) to assist diagnosis. PSG determines whether a person has apnea syndrome with multidimensional data such as brain waves, heart rate, and blood oxygen saturation. In this paper, we have presented a method of recognizing OSAHS, which is convenient for patients to monitor themselves in daily life to avoid delayed treatment. Firstly, we theoretically analyzed the difference between the snoring sounds of normal people and OSAHS patients in the time and frequency domains. Secondly, the snoring sounds related to apnea events and the nonapnea related snoring sounds were classified by deep learning, and then, the severity of OSAHS symptoms had been recognized. In the algorithm proposed in this paper, the snoring data features are extracted through the three feature extraction methods, which are MFCC, LPCC, and LPMFCC. Moreover, we adopted CNN and LSTM for classification. The experimental results show that the MFCC feature extraction method and the LSTM model have the highest accuracy rate which was $87 \%$ when it is adopted for binary-classification of snoring data. Moreover, the AHI value of the patient can be obtained by the algorithm system which can determine the severity degree of OSAHS.

\section{Introduction}

Obstructive apnea hypopnea syndrome (hereinafter called OSAHS) not only leads to poor sleep quality but also leads to chronic hypoxemia, hypercapnia, and even high-grade central nervous system dysfunction lesions, which brings great negative impact to people. Therefore, in order to analyze the reason and sum up the diagnosis method and response treatment policy of OSAHS, more and more researchers are devoted to the research of the disease [1-3]. In clinic practice, polysomnography (hereinafter called PSG) is used to assist doctors in diagnosing OSAHS. PSG obtains multidimensional data such as heart rate, brain waves, chest vibration, blood oxygen saturation, breathing, and snoring. AHI (Apnea Hypopnea Index) value of the patient can be obtained after these data are fused with a certain algorithm and weights by monitoring the patient's breathing throughout the night $[4,5]$, which means the number of apnea index per hour, hypopnea index and obstructive pause, central pause, and mixed pause. However, there are two practical problems with PSG: 1. PSG requires professional medical personnel to operate, and the users must lie in the hospital for monitoring; 2. PSG affects the quality of sleep because the user needs to plug in the corresponding equipment in many parts of the body during use. If there is a technology that does not rely on large medical equipment and is comfortable for patients in daily use, it can not only improve the experience of patients when they are monitored but also help doctors more accurately grasp the long-term clinical performance of patients.

In recent years, scholars have proposed a variety of OSAHS disease discrimination techniques based on various symptom characteristics. Among them, A. Garde used the visual midpoint (radius and angle) distribution characteristics of $\mathrm{SpO}_{2}$ signals to distinguish OSAHS symptoms [6]; Kim used the patient's breathing sound signal to develop a classification of OSAHS severity model [7]; Volak made preliminary judgments on OSAHS through image 
recognition of children's dental features [8]; Castillo-Escario et al. develop an algorithm for detecting silence events and classifying them into apneas and hypopneas [9]. The current medical research reports show that the clinical apnea syndrome events manifestations of an adult are as follows $[10,11]$. Snoring is loud and often be interrupted by an apnea event that lasts for about $10 \mathrm{~s}$; then, a faint snoring sound appears during the incident. After the incident, the patient suffered from gasping, accompanied by loud snoring. The reason for the formation of OSAHS disease is the blockage of the internal cavity of the nose of patients initiated by the patient's oral and nasal diseases such as rhinitis or pharyngitis [12]. In general, snoring is the form of expression of OSAHS. In the study of snoring, the researchers first studied the technique of extracting snoring sounds from breathing sounds. For example, the nonlinear classification algorithm to identify snoring sounds was studied by Ankishan [13]; Lim proposed a snoring recognition method based on RNN $[14,15]$. The study of OSAHS recognition based on snoring has also been proposed after the effective extraction of snoring signals: After extracting the timedomain features of snoring after apnea events, Temrat et al. judged the severity degree of OSAHS through distinguishing different types of snoring by the leave-one-out cross-validation technique [16]. However, the time domain features such as zero-crossing rate (ZCR), energy entropy (EE), and integrated electromyography (IEMG) extracting snoring from background noise in this paper have two problems: (1) The similar features of some audio data are not easy to be distinguished; (2) The feature dimension is too less. Therefore, in order to extract better features, researchers also introduced neural network classification methods into snoring recognition. The advantage of detecting snoring features based on deep learning is that the neural network can automatically extract features. For example, Takahiro Emoto classified snoring data related to OSAHS (SNR) based on ANN. Unfortunately, due to the limitation of the classification effect of ANN, its accuracy result can only reach $75 \%$ [17]. Moreover, the data used in this experiment is not obtained based on the test result data output in PSG which is currently the standard diagnostic procedure for obstructive sleep apnea (hereinafter called OSA) [18] but artificially annotated data by listening to the sound. Therefore, the reliability of the experiment could not be verified by the most reliable device: PSG. In addition, B. Daurai and P. Nayak detected apnea events by using three dimensions of the chest cavity, abdomen, and respiratory airflow. The equipment used for obtaining data is inconvenient to wear and may affect the quality of sleep of users [19]. In this paper, we presented a method for automatic recognition of OSAHS based on snoring sounds classification by a neural network model. Our method only used snoring sounds for recognition. Therefore data of patients could be easily collected by recording equipment. Firstly, the snoring sounds related to apnea events and nonapnea event-related snoring sounds (see Section 2.2) are identified. Secondly, the severity of the apnea event was judged by the result of snoring sound recognition. Each snoring data is converted from the time domain to the frequency domain. The MFCC features are calculated, and then the MFCC features are used as the input of a LSTM model for binary classification. The experiments result indicates that our method could realize a recognition with high accuracy.

\section{Materials and Methods}

2.1. Flow of the Proposed Method. The algorithm flow proposed in this paper is shown in Figure 1, which is divided into two steps: feature extraction and classification. After MFCC feature extraction, snoring audio data were inputted into the CNN/LSTM neural network for binary classification, and then the algorithm outputted the recognition result.

2.2. Feature Extraction. Figure 2 is the data derived from PSG. The results show the multidimensional data such as pulse and oxygen saturation during patient monitoring. As shown in the figure, an obstruction pause event occurs between $23: 01: 10-23: 01: 25$. The blocking pause event is obtained by multidimensional data fusion. From the dimension data of snoring, the apnea event is accompanied by a very weak snoring sound, which is marked with purple in Figure 2. The snoring that appeared after the apnea event is marked with blue. Snoring sounds that occur during apnea events and after apnea events are recorded as snoring sounds related to apnea events, which are called abnormal snoring. The remaining snoring sounds related to nonapnea events are called normal snoring sounds. In order to identify the normal snoring and abnormal snoring, we firstly extracted features of snoring signals using three different feature extraction methods which are MFCC, LPCC, and LPMFCC. The flow of feature extraction is given in Figure 3.

2.2.1. MFCC. MFCC (Mel Frequency Cepstral Coefficient) is a feature inspired by the event that different human ears have different hearing sensitivity to sound waves which have different frequencies. MFCC is currently widely used in the field of audio recognition. Preemphasis, framing, and windowing pretreatment are performed in the time domain before feature extraction [20]. Preemphasis is to pass the speech signal through a high-pass filter in order to compensate for the loss of high-frequency components and improve the high-frequency components. Frame is to gather $\mathrm{N}$ sampling points into a set unit. The purpose is to make the parameters between one frame and another frame transition smoothly. Windowing is to multiply each frame by Hamming window, which is to reduce signal characteristics leak in the frequency domain. After pretreatment, the signal is converted to the frequency domain by Fourier transform and the power spectrum is calculated. Then, the Mel-scale triangular filter bank is used to smooth the frequency spectrum instead of avoiding the characteristic parameters that are affected by the pitch of the speech. Finally, we calculate the log energy and MFCC coefficient of each filter bank output [21]. As shown in Equation (1) and Equation (2), the log energy $s(m)$ output by each filter bank is obtained by Equation (1), where $m$ represents the number of 


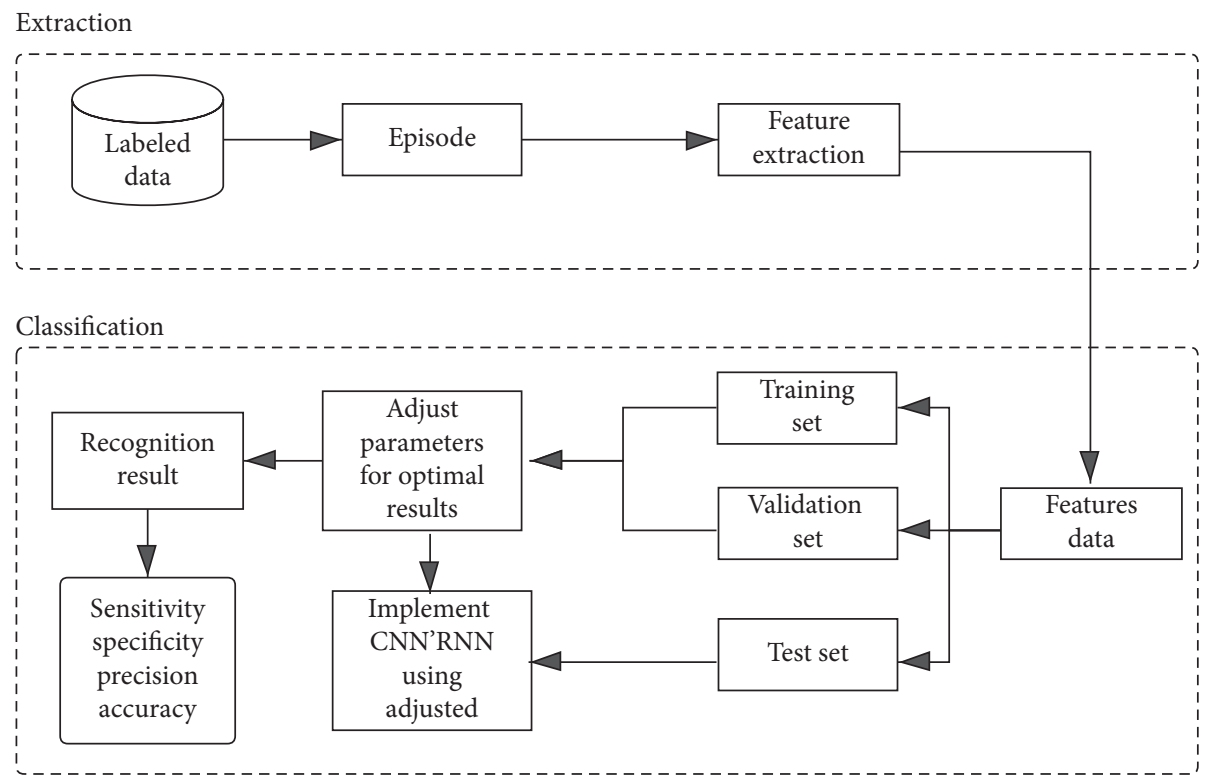

Figure 1: The flow of the proposed method.

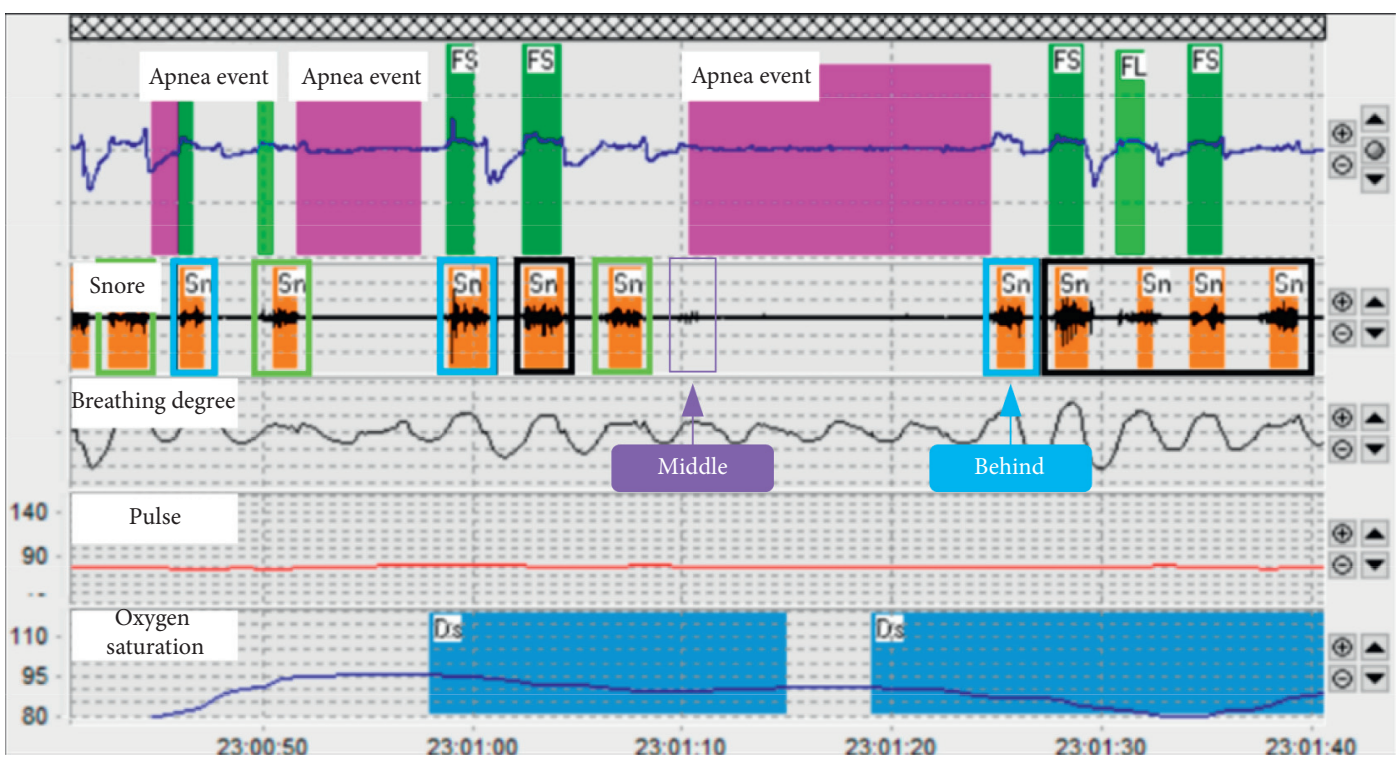

Figure 2: Data presented on PSG.

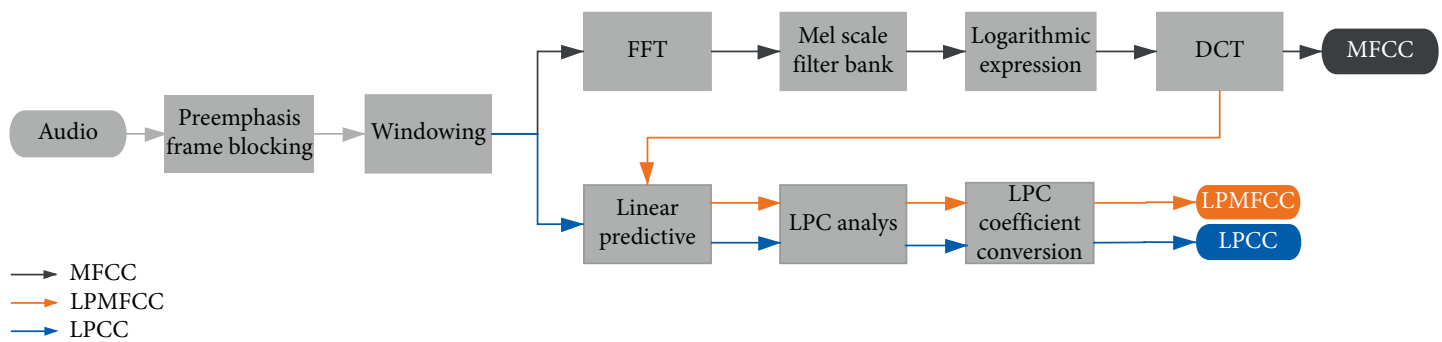

Figure 3: Three methods of feature extraction.

filters, $k$ represents the number of Fourier transform points, $n$ represents the order of MFCC coefficients, $\mathrm{Xa}(k)$ represents the power of the speech signal spectrum obtained by performing fast Fourier transform of each frame signal and taking the modulus square; $H(k)$ represents the frequency response of the energy spectrum obtained by the triangular 
filter; the MFCC coefficient $C(n)$ is obtained based on DCT (discrete cosine transform) [21]:

$$
\begin{aligned}
& s(m)=\ln \left(\sum_{k=0}^{N-1}|X a(k)|^{2} H(k)\right), \quad 0 \leq m \leq M, \\
& C(n)=\sum_{m=0}^{N-1} s(m) \cos \left(\frac{\pi n(m-0.5)}{m}\right), \quad n=1,2, \ldots, L .
\end{aligned}
$$

Each piece of snoring audio was divided into frames by $0.03 \mathrm{~s}$ length of frame and $0.01 \mathrm{~s}$ shift of frame, the extracted feature dimension of which is $298 * 40$.

2.2.2. LPCC. In order to obtain the basic parameters of speech signals, LPCC has become one of the main technologies to estimate the parameters of speech signals. The algorithm of LPCC is shown in Figure 1, except for the same pretreatment as shown in Section 2.2.1, the signal undergoes the current prediction model to calculate the LPC coefficients, and then is converted into LPCC coefficients in the form of the spectrum by cepstrum, $V(z)$ is the channel transfer function. $G$ is the gain of the filter, $a_{k}$ is the set of known linear regression coefficients (LPC) autoregressive coefficients, and $p$ is the order of the all-pole filter. The LPC coefficients obtained by the autocorrelation method ensure the stability of the system so that the channel model transferred function corresponding to the following Equation (3) has a minimum phase [22]. Equation (4) can deduce the recursive relationship between the cepstral $c(n)$ of the speech signal and the LPC coefficient, where $c(1)$ is the DC component and reflects the spectral energy, and its value does not affect the spectral shape. The second formula is used when the number of LPCC coefficients is not greater than the number of LPC coefficients, and the third formula is used when the number of LPCC coefficients is greater than the number of LPC coefficients:

$$
\begin{gathered}
V(z)=\frac{G}{1-\sum_{k=1}^{p} a_{k}+z^{-k}}, \\
\left\{\begin{array}{l}
c(n)=a_{1} \\
c(n)=a_{n}+\sum_{k=1}^{n-1}\left(1-\frac{k}{n}\right) a_{k} c(n-k), \quad 1<n<p \\
c(n)=\sum_{k=1}^{p}\left(1-\frac{k}{n}\right) a_{k} c(n-k), \quad n>p
\end{array}\right.
\end{gathered}
$$

2.2.3. $L P M F C C$. The LPMFCC feature parameters are based on LPC. The process obtained by calculating the Mel Cepstrum of LPC is shown in formula (5). Firstly, the LPC coefficients are subjected to Fourier transform, and then the LPC coefficients are obtained through DFT Discrete spectrum $X_{a}(k)$. Secondly, the square of the spectrum amplitude is calculated to obtain the discrete energy spectrum
$X_{a}(k)^{2}$. Among them, $\mathrm{N}$ represents the number of Fourier transform points. Thirdly, a set of Mel-scale triangular filters are used to filter the discrete energy spectrum, and then the output results are subjected to logarithm operation to obtain the log energy $Z_{a}(m)$, as shown in Equation (6), where $H_{m}(k)(0 \leq \mathrm{m} \leq M)$ is several band-pass filters, and $M$ is the number of filters. Finally, the above logarithmic energy is calculated by discrete cosine transform, and a new characteristic parameter LPMFCC is obtained:

$$
\begin{aligned}
& X_{a}(k)=\sum_{n=0}^{N-1} x_{a}(n) e^{-(j 2 p n k / N)}, \quad 0 \leq k \leq N-1, \\
& Z_{a}(m)=\ln \left(\sum_{k=0}^{N-1}\left|X_{a}(k)\right|^{2} H_{m}(k)\right), \\
& C_{a}(n)=\sum_{m=0}^{M-1} Z_{a}(m) \cos \left[\frac{p n(m+(1 / 2))}{M}\right], \quad 0 \leq m \leq M .
\end{aligned}
$$

\subsection{Model Recognition}

2.3.1. CNN-Based Audio Recognition. Research on convolutional neural networks originated in the late 19th century. Since 2012, due to breakthroughs in hardware and algorithms, both image recognition and audio recognition have made leaps and bounds [23-25]. In the field of audio recognition, Google proposed the CNN model in 2017 to identify keywords [26]. The general convolutional neural network structure usually includes a convolutional layer, a fully connected layer, and a pooling layer. The CNN model used in this article consists of a three-layer $\mathrm{CNN}$ structure, and the extraction result of each CNN convolutional layer is activated and connected to the Relu activation function. The max-pooling layer is finally connected to the fully connected layer to map the distributed features to the sample label space (see Figure 4), and the model parameter settings are shown in Table 1. Considering that the input MFCC features are much less complex than the image data features in image recognition, it is not necessary to adopt very deep neural networks for recognition. If the structure of the recognition model is too deep, it will result in problems such as overfitting and excessive calculation. We will conduct a comparative experiment with 3-layer CNN and 5-layer CNN to verify this viewpoint in Section 3.4, and the construction of 3-layer CNN and 5-layer CNN are shown in Tables 1 and 2.

2.3.2. RNN-Based Audio Recognition. This paper also uses a recurrent neural network (RNN) model for comparing with $\mathrm{CNN}$ in classification performance. RNN is usually used to process a lot of sequence data $\left\{x_{1}, x_{2}, \ldots x_{\mathrm{t}}\right\}$ and is widely used in natural language processing (NLP), speech recognition, translation, and so on. Unlike CNN, RNN has a memory function for time series, which can capture the connection and difference between the characteristics of this time point and the previous time point. RNN can be concluded as 


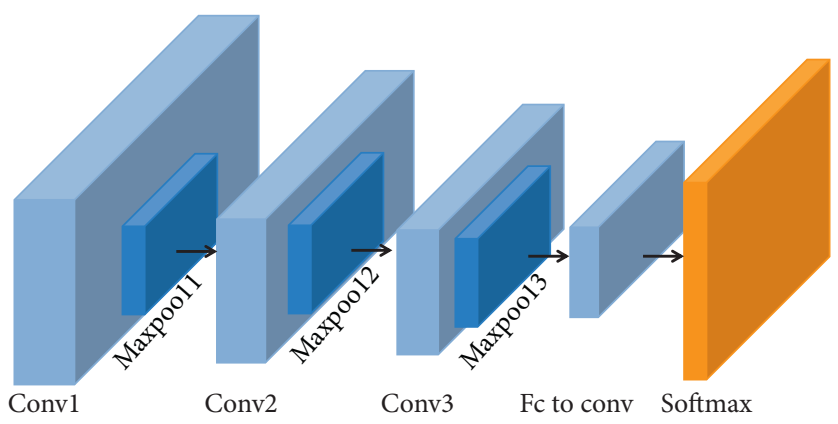

FIGURE 4: CNN architecture used in this paper.

Table 1: Parameters of our 3 layers CNN.

\begin{tabular}{lcccc}
\hline Block & Layer & Filter size & Filters (layer number) & Stride (step number) \\
\hline \multirow{2}{*}{ Conv1 block } & Conv1 maxPool1 & $\begin{array}{c}20 * 8 \\
2 * 2\end{array}$ & 64 & 2 \\
\hline \multirow{2}{*}{ Conv2 block } & \multirow{2}{*}{ Conv2 maxPool2 } & $\begin{array}{c}10 * 4 \\
2 * 2\end{array}$ & 2 \\
\hline \multirow{2}{*}{ Conv3 block } & \multirow{2}{*}{ Conv3 maxPool3 } & $5 * 2$ & 64 & 1 \\
& & $2 * 2$ & 64 & 2 \\
\hline
\end{tabular}

TABle 2: Parameters of our 5 layers CNN.

\begin{tabular}{llccc}
\hline Block & Layer & Filter size & Filters (layer number) & Stride (step number) \\
\hline \multirow{2}{*}{ Conv1 block } & Conv1 maxPool1 & $\begin{array}{c}20 * 8 \\
2 * 2\end{array}$ & 64 & 2 \\
\hline \multirow{2}{*}{ Conv2 block } & Conv2 maxPool2 & $\begin{array}{c}10 * 4 \\
2 * 2\end{array}$ & 2 \\
\hline \multirow{2}{*}{ Conv3 block } & \multirow{2}{*}{ Conv3 maxPool3 } & $5 * 2$ & 64 & 1 \\
\hline \multirow{2}{*}{ Conv4 block } & \multirow{2}{*}{ Conv4 maxPool4 } & $2 * 2$ & 64 & 1 \\
\hline \multirow{2}{*}{ Conv5 block } & \multirow{2}{*}{ Conv5 maxPool5 } & $2 * 2$ & 64 & 2 \\
\hline
\end{tabular}

ordinary RNN and special RNN. Special RNN refers to replacing ordinary short-term memory network unit (LSTM) or gated cycle unit (GRU) with ordinary RNN unit. In this paper, the LSTM unit is used for sequence identification. As shown in Figure 5(a), from left to right in a LSTM unit structure are the forget gate, the input gate, and the output gate. The output gate controls the output of information and filters the information to be output.

The expression at time $t$ is shown in formulae (8) to (13). $x_{t}$ represents the input information, $h_{t-1}$ and $h_{t}$, respectively, represent the hidden coefficient output of the previous LSTM unit and the new LSTM unit. $C_{t-1}$ and $C_{t}$ represent the information output by the previous unit and the new information output by the unit, $f_{t}$ represents the information that the unit selectively forgets, which is multiplied with the weight $W_{f}$ and adds to the coefficient $b_{f} . i_{t}$ and $C_{t}^{\prime}$ represent the memory information, and then are merged into $C_{t}$, which represents the final output state shown in formula (11). $O_{t}$ represents the state of the three gates. Then $h_{t}$ is obtained by the multiplication of $O_{t}$ and $\tanh \left(c_{t}\right)$.
LSTM can make up for the shortcomings of ordinary RNN's short memory and uncontrollable storage content. The operation parameters of LSTM are less than the CNN parameters mentioned above, and the operation is faster, which helps avoid the gradient disappearance and explosion problems in typical RNN [27]. The RNN structure model is shown in Figure 5(b). According to the input audio spectrogram, the display dimension is $t * f=298 * 40$ (where $t$ represents time and $f$ represents frequency). The input data $x_{\mathrm{t}}$ is input to the No.t RNN unit with the hidden unit $h_{t-1}$ of the time frame output. Since one data input frame number is 298 , the RNN model has 298 LSTM units, $x_{0} \sim x_{297}$ represents the input feature, and $y$ represents the output result (see Figure 5(b)):

$$
\begin{aligned}
f_{t} & =\theta\left(W_{f} \cdot\left[h_{t-1}, x_{t}\right]+b_{f}\right), \\
i_{t} & =\theta\left(W_{i} \cdot\left[h_{t-1}, x_{t}\right]+b_{i}\right), \\
C_{t}^{\prime} & =\tanh \left(W_{c} \cdot\left[h_{t-1}, x_{t}\right]+b_{c}\right),
\end{aligned}
$$




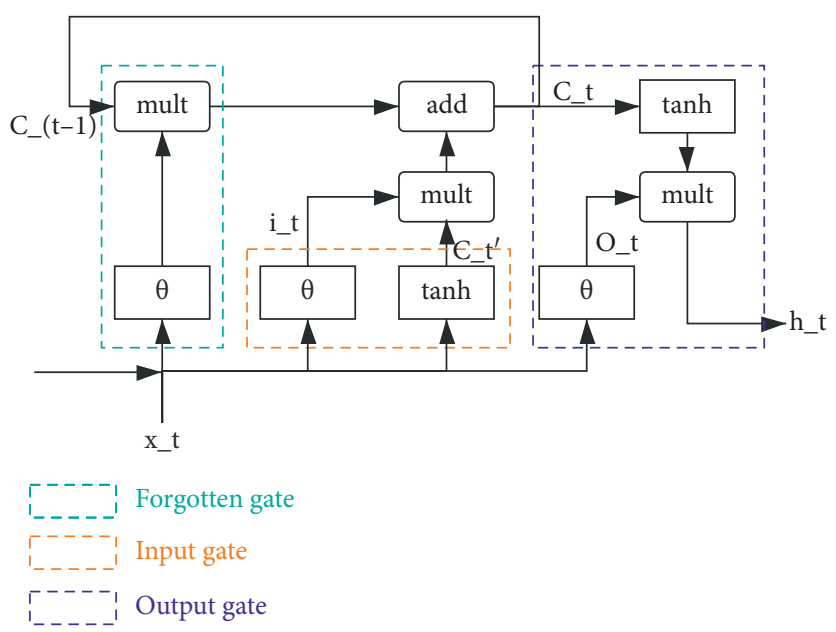

(a)

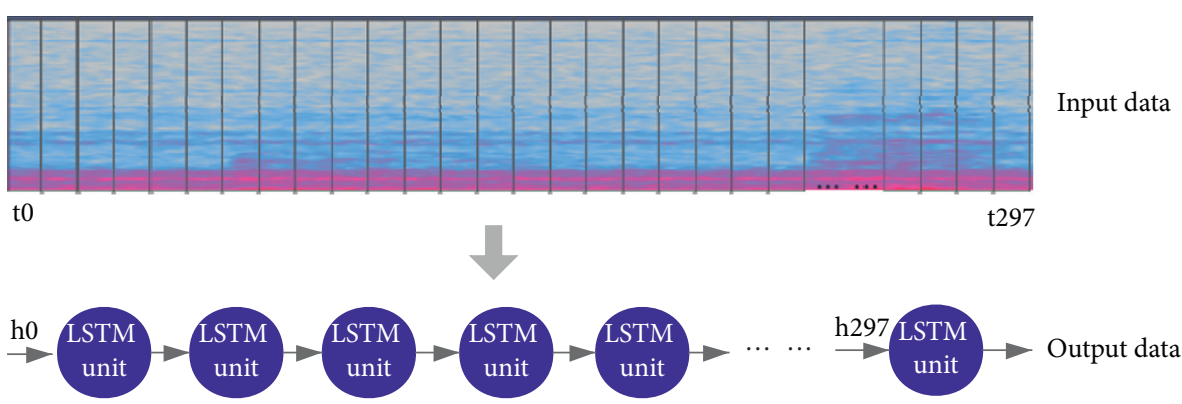

(b)

Figure 5: RNN-based audio recognition. (a) LSTM cell structure. (b) LSTM neural network model.

$$
\begin{aligned}
& C_{t}=f_{t} * C_{t-1}+i_{t} * C_{t}^{\prime}, \\
& O_{t}=\theta\left(W_{o} \cdot\left[h_{t-1}, x_{t}\right]+b_{o}\right), \\
& h_{t}=O_{t} * \tanh \left(c_{t}\right) .
\end{aligned}
$$

\section{Results and Discussion}

3.1. Database. In our experiments, sleep sounds are collected from 32 volunteers (including 16 normal people and 16 OSAHS patients) through a microphone for a whole night $(8 \mathrm{~h})$ at a sampling frequency of $16 \mathrm{KHz}$. The device can be used at home. Snoring sounds were extracted from breathing sounds and background noise through the endpoint detection technology [27], each case of which lasting $3 \mathrm{~s}$. The training samples consisted of randomly selected 16 volunteers' snoring sounds (5 normal people and 11 OSAHS patients). We classified snoring data from OSAHS patients' sleeping sounds in the whole night according to the time period displayed by the PSG picture into four categories for ready: (1) Snoring data before each apnea event; (2) Snoring data in each apnea event; (3) Snoring data after each apnea event; (4) The other snoring data in OSAHS patients' snoring data in a whole sleeping night.

3.2. Experimental Evidence of Data Selection. In order to verify that the snoring data which during the apnea events and behind of the apnea events have obvious characteristics differences from normal snoring data extracted from 5 normal people while sleeping at night [20], we implemented four sets of comparative experiments (see Table 3): (1) Snoring data before, during, and after apnea events and normal snoring data extracted from normal people's sleeping sounds while at night as two-class sample sets; (2) Snoring data during and after apnea events and normal snoring data extracted from normal people's sleeping sounds in the whole night as two-class sample sets; (3) Snoring data before apnea events and normal snoring data extracted from normal people's sleeping sounds in the whole night as twoclass sample sets; (4) All of the snoring data extracted from OSAHS patients and normal snoring data extracted from normal people's sleeping sounds in the whole night as twoclass sample sets. Each set of data is about 10000 cases and the number ratio of the two categories is $1: 1$.

Conclusions can be drawn from this experiment: the data characteristics of snoring data during the apnea events and behind of the apnea events have the most obvious differences from normal snoring data extracted from normal people's whole sleeping night.

3.3. Experiment. Based on the above experimental evidence in Section 3.2, the positive sample (normal snoring) is the data from the 5 normal people's whole night snoring, and the negative sample (abnormal snoring) is the data from the 
TABLE 3: Results of four groups of experiments.

\begin{tabular}{|c|c|c|c|c|}
\hline Test group (number) & Sensitivity & Specificity & Precision & Accuracy \\
\hline 1 & 0.75 & 0.75 & 0.50 & 0.60 \\
\hline 2 & 0.83 & 0.84 & 0.82 & 0.82 \\
\hline 3 & 0.42 & 0.53 & 0.43 & 0.48 \\
\hline 4 & 0.40 & 0.50 & 0.40 & 0.45 \\
\hline
\end{tabular}

TABle 4: Data distribution.

\begin{tabular}{|c|c|c|c|}
\hline Subjects & \multicolumn{2}{|c|}{16 people } & 16 people \\
\hline Class & Training set & Validation set & Test set \\
\hline Normal snoring(case) & 5156 & 516 & 2065 \\
\hline Abnormal snoring (case) & 5156 & 516 & 2065 \\
\hline
\end{tabular}

OSAHS patients' whole night snoring, which were taken from the apnea event's middle and behind (see Figure 2). The snoring data of the remaining 16 volunteers were used to test the generalization performance of the model (see Table 4).

3.4. Environment. The experiment in this article is operated in the ubuntu 5.4.0 environment, rtx2048 graphics card. The model is built using TensorFlow 2.0 framework. The RNN and CNN network parameters are shown in Section 2.3.

3.5. Evaluation. The experimental results are evaluated by 5 methods which are accuracy, sensitivity, specificity, precision, and F1-score. The expressions of these methods are given by formulae (14) to (18). In these formulas, TP presents the number of positive samples that were actually identified as positive samples; FN presents the number of the positive samples that were identified as the negative samples falsely; TN presents the number of negative samples that were correctly identified as the negative sample; FP presents the number of the negative samples that were identified as the positive sample falsely:

$$
\begin{aligned}
\text { accuracy } & =\frac{T P+T N}{T P+T N+F P+F N}, \\
\text { precision } & =\frac{T P}{T P+F P}, \\
\text { sensitivity } & =\text { recall }=\frac{T P}{T P+F N}, \\
\text { specificity } & =\frac{T N}{T P+F P}, \\
F 1-\text { score } & =\frac{2 * \text { precision } * \text { recall }}{\text { precision }+ \text { recall }},
\end{aligned}
$$

3.6. Comparison of Three Feature Extraction Methods and Three Model Experiment Results. Table 5 shows the accuracy of the model test combined with the three feature extraction methods (MFCC, LPCC, LPMFCC) and the three models (3- layer CNN, 5-layer CNN, and LSTM). A horizontal comparison found that the classification effect of 5-layer $\mathrm{CNN}$ is the same as 3-layer CNN, and LSTM performs better than $\mathrm{CNN}$ in the connection and comparison of time series data features, and LSTM can make up for the shortcomings of ordinary RNNs with a short memory and uncontrollable storage content [27]. As shown in Table 5 and Figure 6, through longitudinal comparison, it is found that the feature extraction effect from the best to the worst are MFCC, LPMFCC, LPCC. This is because the LPCC algorithm has a linear prediction function for time series and can obtain more information from speech recognition [28], while MFCC maps the speech frequency to a nonlinear mel filter bank and converts it to the cepstrum domain [29]. The features extracted from the neighboring frames are almost independent and suitable for consonant recognition. The snoring sound produced by a person during sleep is caused by the blockage of the upper respiratory tract of the person due to some reason (rhinitis or pharyngitis or even cerebral nerves [30]), which causes the airflow to hit the soft tissue and generate large vibrations, like consonant. Therefore, the MFCC is more suitable for extracting a feature from snoring data in this paper.

3.7. Discussion of the Application. According to the snoring sounds data related to apnea events in 11 OSAHS patients and the whole night (about 8 hours) snoring sounds of 5 normal subjects as the test set, the LSTM model has a better classification effect than CNN, calculating the number of two types of snoring sounds from 16 volunteers' whole night after they were entered into the stored LSTM model for binary classification. Finally, calculating the AHI value according to the definition of AHI (number of sleep apnea per hour). As shown in formula (19), where $A B$ represents the number of snoring sounds related to the apnea event (Abnormal snoring) recognized by the system, 2 represents 1 case of middle snoring sound and 1 case of posterior snore sound, and $\mathrm{SH}$ represents the length of sleep throughout the night. According to the AHI value, the severity of OSAHS patients can be obtained, which is divided into slight $(5<\mathrm{AHI} \leq 15)$, moderate $(15<\mathrm{AHI} \leq 30)$, and serious $(\mathrm{AHI}>30)[31]$. 
TABLE 5: Accuracy of three feature extraction methods and three models.

\begin{tabular}{|c|c|c|c|c|}
\hline Feature extraction method & Evaluation & 3 layers-CNN & 5 layers-CNN & LSTM \\
\hline \multirow{5}{*}{ MFCC } & Sensitivity & 0.83 & 0.82 & 0.84 \\
\hline & Specificity & 0.84 & 0.81 & 0.91 \\
\hline & Precision & 0.82 & 0.84 & 0.91 \\
\hline & Accuracy & 0.82 & 0.85 & 0.87 \\
\hline & F1-score & 0.82 & 0.83 & 0.87 \\
\hline \multirow{5}{*}{ LPMFCC } & Sensitivity & 0.58 & 0.6 & 0.67 \\
\hline & Specificity & 0.64 & 0.67 & 0.72 \\
\hline & Precision & 0.62 & 0.64 & 0.71 \\
\hline & Accuracy & 0.61 & 0.63 & 0.69 \\
\hline & F1-score & 0.6 & 0.62 & 0.69 \\
\hline \multirow{5}{*}{ LPCC } & Sensitivity & 0.54 & 0.56 & 0.56 \\
\hline & Specificity & 0.6 & 0.6 & 0.62 \\
\hline & Precision & 0.58 & 0.58 & 0.6 \\
\hline & Accuracy & 0.57 & 0.58 & 0.59 \\
\hline & F1-score & 0.56 & 0.57 & 0.58 \\
\hline
\end{tabular}

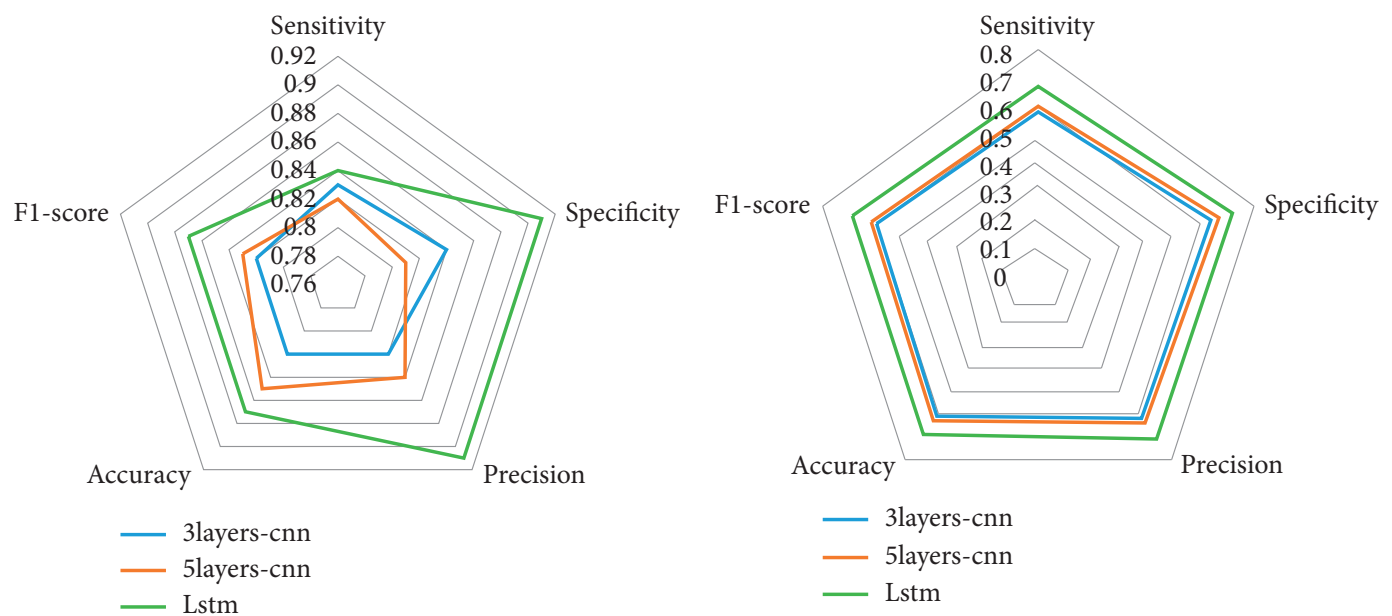

(a)

(b)

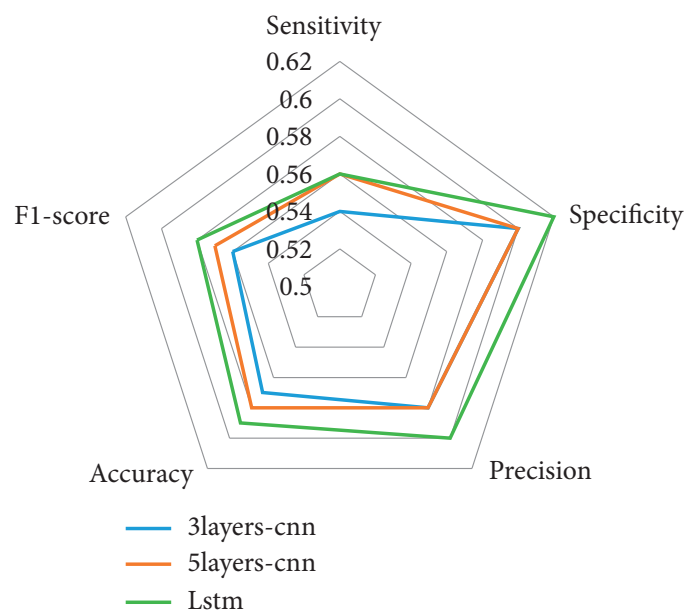

(c)

Figure 6: Accuracy of three feature extraction methods and three models. (a) Accuracy of MFCC method. (b) Accuracy of LPMFCC method. (c) Accuracy of LPCC method. 
TABLE 6: Comparison of the OSAHS degree of the test result and the OSAHS degree data output in PSG.

\begin{tabular}{|c|c|c|c|c|c|}
\hline Subject number & AHI (PSG) & AHI (test) & SQRT-AHI & Degree (PSG) & Degree (test) \\
\hline 01 & 5 & 2 & 0.75 & 0 & 0 \\
\hline 02 & 4.7 & 0.37 & 1.08 & 0 & 0 \\
\hline 03 & 1.7 & 6 & 1.07 & 0 & 1 \\
\hline 04 & 2.8 & 1.7 & 0.28 & 0 & 0 \\
\hline 05 & 1.5 & 1.7 & 0.05 & 0 & 0 \\
\hline 06 & 41.9 & 50.3 & 2.1 & 3 & 3 \\
\hline 07 & 25.3 & 28.1 & 0.7 & 2 & 2 \\
\hline 08 & 7.2 & 8 & 0.2 & 1 & 1 \\
\hline 09 & 31.7 & 31.4 & 0.075 & 3 & 3 \\
\hline 10 & 8.6 & 41 & 8.1 & 1 & 3 \\
\hline
\end{tabular}

As shown in Table 6(0 means no OSAHS, 1 means slight, 2 means moderate, and 3 means serious), 10 patients were tested, and the difference between the AHI which was calculated by test data and the AHI obtained by the patient through PSG actually is represented by squared different (SORT-AHI) shown in Table 6. It can be known from the table that the model has better recognition performance for the severity of OSAHS.

$$
A H I=\frac{A B}{2} / S H .
$$

\section{Conclusions}

In this study, the detection of OSAHS was quantified and evaluated using the feature extraction method and deep learning algorithm. We compared the differences in detection accuracy between three feature extraction methods and three neural networks. It is found that the MFCC performs best in feature extraction and the LSTM performs better than the CNN in classification, and the combination of MFCC and LSTM performed the best, in which the accuracy of classification reached 0.87 . In addition, the model can not only judge whether someone else has OSAHS through snoring but also can detect the severity degree by AHI coefficient (see Table 5), and the model has solved the problem that PSG has. However, the accuracy of the system in this study is not very well. We are going to improve the performance of the system by producing a better neural network model in the feature.

\section{Data Availability}

Data are available upon request to the corresponding author.

\section{Conflicts of Interest}

The authors declare that they have no conflicts of interest.

\section{Acknowledgments}

The dataset was supported by the Affiliated Hospital of Hangzhou Normal University. This project was also supported by the grants from Zhejiang Key Research and Development Project (2019C03088) and Zhejiang Province Commonweal Projects (LGG18F010010).

\section{References}

[1] T. Mao, J. Zhang, Y. Qiao et al., "Uncovering synergistic mechanism of Chinese herbal medicine in the treatment of atrial fibrillation with obstructive sleep apnea hypopnea syndrome by network pharmacology," Evidence-Based Complementary and Alternative Medicine, vol. 2019, Article ID 8691608, 13 pages, 2019.

[2] L. Zhou, R. Ouyang, H. Luo et al., "Dysfunction of nrf2-ARE signaling pathway: potential pathogenesis in the development of neurocognitive impairment in patients with moderate to severe obstructive sleep apnea-hypopnea syndrome," Oxidative Medicine and Cellular Longevity, vol. 2018, Article ID 3529709, 15 pages, 2018.

[3] H.-W. Feng, T. Jiang, H.-P. Zhang et al., "Comparisons of thyroid hormone, intelligence, attention, and quality of life in children with obstructive sleep apnea hypopnea syndrome before and after endoscopic adenoidectomy," BioMed Research International, vol. 2015, Article ID 523716, 6 pages, 2015.

[4] E. Z. Osman, J. Osborne, P. D. Hill, and B. W. V. Lee, "Snoring assessment: do home studies and hospital studies give different results?" Clinical Otolaryngology and Allied Sciences, vol. 23, no. 6, pp. 524-527, 1998.

[5] L. Cai, J. Le, X. Ruan, and M. Du, "OSA patient monitoring system based on the Internet of Things framework," in Proceedings of the 2019 4th International Conference on Smart and Sustainable Technologies (Splitech), pp. 12-15, Split, Croatia, August 2019.

[6] A. Garde, P. Dekhordi, C. L. Petersen et al., "Detecting obstructive sleep apnea in children by self-affine visualization of oximetry," in Proceedings of the 2017 39th Annual International Conference of the IEEE Engineering in Medicine and Biology Society (EMBC), pp. 3757-3760, Seogwipo, Republic of Korea, July 2017.

[7] J. Kim, T. Kim, D. Lee, J.-W. Kim, and K. Lee, "Exploiting temporal and nonstationary features in breathing sound analysis for multiple obstructive sleep apnea severity classification," BioMedical Engineering OnLine, vol. 16, no. 1, 6 pages, 2017.

[8] J. Volák, D. Koniar, L. Hargaš, F. Jablončík, N. Sekelová, and P. Durdík, "RGB-D imaging used for OSAS diagnostics," in Proceedings of the 2018 ELEKTRO, pp. 1-5, Mikulov, Czech Republic, May 2018.

[9] Y. Castillo-Escario, I. Ferrer-Lluis, J. M. Montserrat, and R. Jane, "Entropy analysis of acoustic signals recorded with a smartphone for detecting apneas and hypopneas: a comparison with a commercial system for home sleep apnea diagnosis," IEEE Access, vol. 7, pp. 128224-128241, 2019. 
[10] G. B. Salah, K. Abbes, C. Abdelmoula et al., "Obstructive sleep apnea OSA detection through esophageal pressure pes," in Proceedings of the IEEE International Conference on Design \& Test of Integrated Micro \& Nano-Systems (DTS), pp. 1-4, Gammarth-Tunis, Tunisia, April 2019.

[11] A. Yilmaz, M. A. Mutlu, M. Günay et al., "Analysis of portable apnea devices: comparison with polysomnographic(psg) records," in Proceedings of the 2019 27th Signal Processing and Communications Applications Conference (SIU), pp. 1-4, Sivas, Turkey, April 2019.

[12] K. Srividya, M. Hemamalini, K. Balaswathi et al., "Diagnosing the obstructive sleep APNEA from multi parameter values," in Proceedings of the 2018 International Conference on Communication and Signal Processing (ICCSP), pp. 0616-0619, Chennai, India, April 2019.

[13] H. Ankishan and A. T. Tuncer, "A new portable device for the snore/non-snore classification," in Proceedings of the International Conference on Engineering and Technology, Kayseri, Turkey, February 2017.

[14] S. J. Lim, S. J. JangJ. Y. Lim et al., Classification of snoring sound based on a recurrent neural network," Expert Systems with Applications, vol. 123, pp. 237-2451, 2019.

[15] B. Arsenali et al., "Recurrent neural network for classification of snoring and non-snoring sound events," in Proceedings of the 2018 40th Annual International Conference of the IEEE Engineering in Medicine and Biology Society (EMBC), pp. 328-331, Honolulu, HI, USA, July 2018.

[16] P. Temrat, Y. Jiraraksopakun, A. Bhatranand et al., "Suitable feature selection for OSA classification based on snoring sounds," in Proceedings of the 2018 15th International Conference on Electrical Engineering/Electronics, Computer, Telecommunications and Information Technology (ECTICON), pp. 396-399, Chiang Rai, Thailand, July 2018.

[17] T. Emoto, U. R. Abeyratne, K. Kawano, T. Okada, O. Jinnouchi, and I. Kawata, "Detection of sleep breathing sound based on artificial neural network analysis," Biomedical Signal Processing and Control, vol. 41, pp. 81-89, 2018.

[18] L. Wen-Yen, C. Wen-Cheng, S. Tsu-Hui, S. Guang-Ming, L. Chin-Shan, and L. Ming-Yih, "Realization of a CORDICbased plug-in accelerometer module for PSG system in head position monitoring for OSAS patients," Journal of Healthcare Engineering, vol. 2017, Article ID 4728187, 9 pages, 2017.

[19] B. Daurai, P. Nayak, and R. P. Mudhalwadkar, "Sleep physiological parameter measurement for breath, chest and abdomen effort to detect apnea hypopnea," in Proceedings of the 2017 International Conference on Intelligent Computing and Control (I2C2), Coimbatore, India, June 2017.

[20] A. Winursito, R. Hidayat, and A. Bejo, "Improvement of MFCC feature extraction accuracy using PCA in Indonesian speech recognition," in Proceedings of the 2018 International Conference on Information and Communications Technology (ICOIACT), pp. 379-383, Yogyakarta, Indonesia, March 2018.

[21] P. Peng, Z. He, and L. Wang, "."Automatic classification of microseismic signals based on MFCC and GMM-HMM in underground mines," Shock and Vibration, vol. 2019, Article ID 5803184, 9 pages, 2019.

[22] K. Daqrouq and K. Y. Al Azzawi, “Average framing linear prediction coding with wavelet transform for text-independent speaker identification system," Computers and Electrical Engineering, vol. 38, no. 6, pp. 1467-1479, 2012.

[23] H. Yanagisawa, T. Yamashita, and H. Watanabe, "A study on object detection method from manga images using CNN," in Proceedings of the 2018 International Workshop on Advanced
Image Technology (IWAIT), pp. 1-4, Chiang Mai, Thailand, January 2018.

[24] X. Lin, J. Liu, and X. Kang, "Audio recapture detection with convolutional neural networks," IEEE Transactions on Multimedia, vol. 18, no. 8, pp. 1480-1487, 2016.

[25] C. Yang, W. Jiang, and Z. Guo, "Time series data classification based on dual path CNN-RNN cascade network," IEEE Access, vol. 7, pp. 155304-155312, 2019.

[26] O. A. Sercan and M. Kliegl, "Convolutional neural networks for small-footprint keyword spotting," Submitted on 15 Mar 2017 (v1), last revised 4 Jul 2017.

[27] D. Wu, Z. Tao, and Y. Wu, "Speech endpoint detection in noisy environment using Spectrogram Boundary Factor," in Proceedings of the 2016 9th International Congress on Image and Signal Processing, BioMedical Engineering and Informatics (CISP-BMEI), pp. 964-968, Datong, China, October 2016.

[28] T. R. J. Kumari and H. S. Jayanna, "Comparison of LPCC and MFCC features and GMM and GMM-UBM modeling for limited data speaker verification," in Proceedings of the 2014 IEEE International Conference on Computational Intelligence and Computing Research, pp. 1-6, Coimbatore, India, December 2014

[29] S. Misra, T. Das, P. Saha, U. Baruah, and R. H. Laskar, "Comparison of MFCC and LPCC for a fixed phrase speaker verification system, time complexity and failure analysis," in Proceedings of the 2015 International Conference on Circuits, Power and Computing Technologies [ICCPCT-2015], pp. 1-4, Nagercoil, India, March 2015.

[30] V. Koka, S. Baron, D. Abedipour, V. Latournerie, and P. El Chater, "EK sign: a wrinkling of uvula and the base of uvula in obstructive sleep apnea-hypopnea syndrome," Sleep Disorders, vol. 2015, pp. 1-4, 2015.

[31] C. Zheng, H. Song, S. Wang et al., "Serum uric acid is independently associated with risk of obstructive sleep apneahypopnea syndrome in Chinese patients with type 2 diabetes," Disease Markers, vol. 2019, Article ID 4578327, 6 pages, 2019. 